\title{
GrineColocicical cancer Robotic-assisted psoas hitch with ureteral reimplantation
}

Jennifer J Mueller, ${ }^{1,2}$ Mario M Leitao, Jr ${ }^{1,2}$

\section{ABSTRACT}

This video is too large to play in the PDF, please visit the full text version online at: https://ijgc.bmj.com/content/ $29 / 1 / 223$

${ }^{1}$ Gynecology Service, Department of Surgery, Memorial Sloan Kettering New York, USA

${ }^{2}$ Department of Obstetrics and Gynecology, Weill Cornell Medical College, New York City, New York, USA

\section{Correspondence to}

Jennifer J Mueller, Gynecology Service, Department of Surgery, Memorial Sloan Kettering Cancer Center, New York City, NY 10065, USA; muellerj@ mskcc.org

Accepted 1 October 2018 Cancer Center, New York City,

Objective To demonstrate a robotic-assisted psoas hitch with ureteral reimplantation.

Methods We gleaned video footage from a robotic-assisted psoas hitch procedure performed for a patient with an isolated pelvic recurrence of ovarian cancer.

Results We demonstrate trocar placement and a roboticarm docking strategy for pelvic recurrence of ovarian cancer. We also show surgical steps involved in a psoas hitch and reimplantation of a transected ureter into the bladder. Special emphasis is placed on guiding the surgeon using key robotic instruments and materials to optimize the robotic completion of this procedure. Key components of the procedure, including en bloc tumor excision and ureteral transection, are shown. The bladder is placed on traction using the fourth arm, and the avascular planes of dissection, including the space of Retzius and the paravesical spaces, are shown. The bladder is then backfilled to allow the surgeon to determine the ideal placement of the ureteral reimplantation to ensure the anastomosis is tension free. The surgeon then demonstrates where and how to place anchoring sutures from the bladder to the psoas muscle. The ureter is examined to determine where it can be implanted in the bladder with zero tension or angulation, which would compromise function and healing. The ureter is prepared for

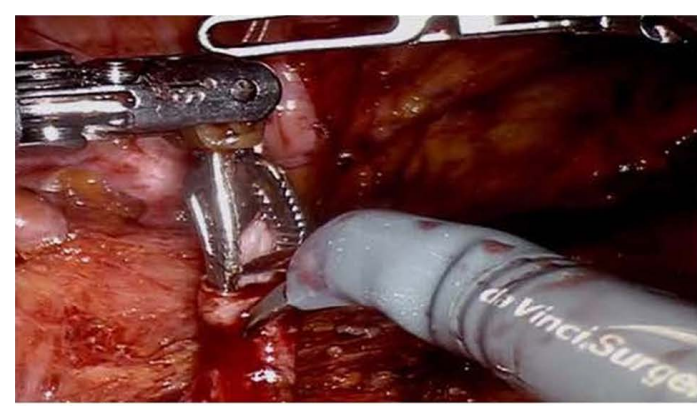

\section{Robotic assisted psoas hitch with ureteral reimplantation} commercial re-use. See rights and permissions. Published by BMJ.

\footnotetext{
To cite: Mueller JJ, Leitao, Jr MM. Int J Gynecol Cancer 2019;29:223.
}

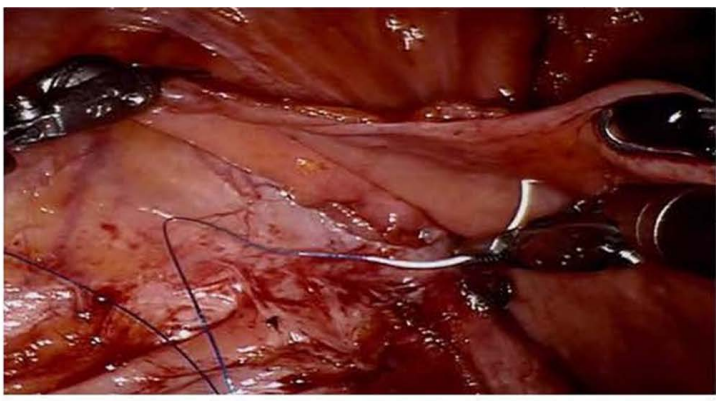

reimplantation, including trimming, tagging, and spatulation. An instrument tie technique is used to implant the ureter into the bladder and a ureteral stent is placed. Robotic-assisted psoas hitch with ureteral reimplantation has been described in the literature. ${ }^{1-4}$

Conclusions Through the use of still photographs and video, we demonstrate the technique of robotic-assisted psoas hitch with ureteral reimplantation.

Competing interests $M M L$ is an ad hoc speaker for Intuitive Surgical. JJM does not have conflicts of interest to disclose.

Provenance and peer review Not commissioned, internally peer reviewed.

\section{REFERENCES}

1. Pugh J, Farkas A, Su LM. Robotic distal ureterectomy with psoas hitch and ureteroneocystostomy: surgical technique and outcomes. Asian J Urol 2015;2:123-7.

2. Singh I. Robotic distal ureterectomy with reimplantation in malignancy: technical nuances. Can J Urol 2009;16467:1-6.

3. Maldonado PA, Slocum PD, Chin K, et al. Anatomic relationships of psoas muscle: clinical applications to psoas hitch ureteral reimplantation. Am J Obstet Gynecol 2014;211:563.e1-563.e6.

4. Madalina AO, Jeremie S, Benoit R, et al. Laparoscopic ureteroneocystostomy with a vesicopsoas hitch in 10 steps. J Minim Invasive Gynecol 2018;25:951.

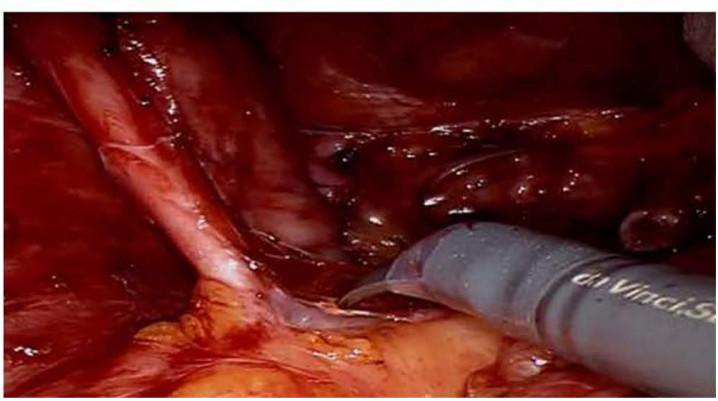

